\title{
Disentangling syntrophy
}

Syntrophic interactions, in which two or more microorganisms cooperate metabolically to metabolize compounds that neither partner can metabolize alone, are common in environmental niches. Zengler and colleagues present a 'multi-omics', systems-based workflow to investigate the details of a syntrophic relationship between two Geobacter species.

Syntrophy that involves interspecies electron transfer is often found in methanogenic environments. Although this is mostly thought to involve interspecies hydrogen transfer, direct interspecies electron transfer (DIET) has been observed in some methanogenic syntrophies. One such laboratory-evolved association involves Geobacter metallireducens and Geobacter sulfurreducens, which can grow syntrophically on ethanol and fumarate using DIET from G. metallireducens to G. sulfurreducens.

The authors updated the genomescale metabolic reconstructions that are available for both partners, to account for the energy metabolism that is involved in extracellular and direct electron transfer. These models were combined to provide an integrated genomic model of syntrophy, introducing a shared metabolite pool component to allow for the exchange of metabolites both between species and with the external environment. The electron transfer flux constraint was defined, which enabled DIET to be modelled. Physiological and transcriptomic data were also added into the mix.

The results confirmed that DIET was the main mode of electron transfer from G. metallireducens to G. sulfurreducens, and showed that — in addition to ethanol oxidation and fumarate reduction - nitrogen fixation by G. sulfurreducens was also essential. Transcriptomic analysis of two mutant strains of G. metallireducens revealed that optimal electron flux via DIET required acetate transfer. To gain an insight into the factors that determine whether interspecies hydrogen transfer or DIET is preferred, the authors replaced G. metallireducens with Pelobacter carbinolicus, which exchanges electrons with G. sulfurreducens by intraspecies hydrogen transfer rather than by DIET. Comparative analysis of the different modes of electron transfer identified several factors that influence which mode of transfer is preferred, including the local availability of protons.

Finally, the authors investigated the adaptations that occured during syntrophic growth and found that adaptation involved genomic and transcriptomic changes in the dominant partner, G. sulfurreducens, whereas only transcriptomic changes were observed in G. metallireducens. This led the authors to suggest that, in syntrophic associations, the electronaccepting partner may undergo metabolic streamlining to favour the most efficient mode of electron transfer.

Sheilagh Molloy

ORIGINAL RESEARCH PAPER Nagarajan, H. et al. Characterization and modelling of interspecies electron transfer mechanisms and microbial community dynamics of a syntrophic association. Nature Commun. http://dx.doi.org/10.1038/ ncomms3809 (2013)

FURTHER READING Zengler, K. \& Palsson, B. O. A roadmap for the development of community systems (CoSy) biology. Nature Rev. Microbiol. 10, 366-372 (2012) 